\title{
KHINTCHINE'S THEOREM AND TRANSFERENCE PRINCIPLE FOR STAR BODIES
}

\author{
M. M. DODSON AND S. KRISTENSEN
}

\begin{abstract}
Analogues of Khintchine's Theorem in simultaneous Diophantine approximation in the plane are proved with the classical height replaced by fairly general planar distance functions or equivalently star bodies. Khintchine's transference principle is discussed for distance functions and a direct proof for the multiplicative version is given. A transference principle is also established for a different distance function.
\end{abstract}

\section{INTRODUCTION}

A star body $S$ in Euclidean space $\mathbb{R}^{n}$ is defined as an open set containing the origin and for which given any $x \in \mathbb{R}^{n}$, there exists a $t_{0} \in(0, \infty]$ such that for $t<t_{0}, t x \in S$ and for $t>t_{0}, t x \notin S$ (see [8]). To such sets, one can associate a continuous distance function $F: \mathbb{R}^{n} \rightarrow[0, \infty)$ such that for any $x \in \mathbb{R}^{n}$ and any $t \geq 0, F(t x)=t F(x)$. The open star body $S$ may be expressed as the set of points satisfying $F(x)<1$. Conversely, we can associate an open star body to any distance function by this relation. Some authors consider only star bodies symmetric about the origin, but this restriction is not needed here and we consider the more general case. However, we restrict ourselves principally to the planar case $(n=2)$, as in higher dimensions the number of cases to be considered proliferates and geometry becomes very complicated.

Examples of planar distance functions include the height on $\mathbb{R}^{2}$ given by $\left|\left(x_{1}, x_{2}\right)\right|_{\infty}$ $=\max \left\{\left|x_{1}\right|,\left|x_{2}\right|\right\}$, where the associated star body is a square. Another example is the function given by $\left(x_{1}, x_{2}\right) \mapsto \sqrt{\left|x_{1}\right|\left|x_{2}\right|}$ where the associated star body is bounded by the hyperbola $x_{2}=1 / x_{1}$ and its mirror image $x_{2}=-1 / x_{1}$. Such symmetric star bodies and their relationship to lattices have been studied extensively. We refer to 8 for an excellent introduction.

In this paper, we study the relationship between planar star bodies and metric Diophantine approximation. Let $F: \mathbb{R}^{2} \rightarrow[0, \infty)$ be a distance function and let $\psi: \mathbb{N} \rightarrow(0, \infty)$ be a function with $q \psi(q)$ non-increasing. For convenience we study the set

$$
\begin{aligned}
W(F ; \psi)= & \left\{x \in \mathbb{T}^{2}: F(x-p / q)<\psi(q) \text { for some } p \in \mathbb{Z}^{2}\right. \\
& \text { for infinitely many } q \in \mathbb{N}\} \\
= & \bigcap_{N=1}^{\infty} \bigcup_{q=N}^{\infty} \bigcup_{p \in \mathbb{Z}^{2}}\left\{x \in \mathbb{T}^{2}: F(q x-p)<q \psi(q)\right\},
\end{aligned}
$$

where $\mathbb{T}^{2}$ denotes the two-torus $\left\{\left(x_{1}, x_{2}\right) \in \mathbb{R}^{2}: 0 \leq x_{1}, x_{2}<1\right\}$. When $F$ is the height $|\cdot|_{\infty}, W(F ; \psi)$ is the set of simultaneously $\psi$-approximable points. When $F$ is the function

2000 Mathematics Subject Classification. 11J83, 11H16.

Key words and phrases. Diophantine approximation; distance functions; metric theory. 
$\left(x_{1}, x_{2}\right) \mapsto \sqrt{\left|x_{1}\right|\left|x_{2}\right|}, W(F ; \psi)$ is the set of multiplicatively $\psi$-approximable points. In both of these special cases, we can restrict $p$ to those points with $0 \leq|p|<q$ without loss of generality. As we shall see, such restrictions are not natural in the general case.

Let $|E|$ denote the Lebesgue measure of $E \subseteq \mathbb{R}^{2}$. Khintchine's Theorem (see e.g. [7]), which asserts that when $q \psi(q)$ is decreasing,

$$
\left|W\left(|\cdot|_{\infty} ; \psi\right)\right|= \begin{cases}0 & \text { if } \sum_{q=1}^{\infty} q^{2} \psi(q)^{2}<\infty \\ 1 & \text { if } \sum_{q=1}^{\infty} q^{2} \psi(q)^{2}=\infty\end{cases}
$$

was extended by Gallagher [12] who established a general theorem implying (2) and the following:

$$
\left|W\left(\left(x_{1}, x_{2}\right) \mapsto \sqrt{\left|x_{1}\right|\left|x_{2}\right|} ; \psi\right)\right|= \begin{cases}0 & \text { if } \sum_{q=1}^{\infty} q^{2} \psi(q)^{2} \log \left(q^{-1} \psi(q)^{-1}\right)<\infty \\ 1 & \text { if } \sum_{q=1}^{\infty} q^{2} \psi(q)^{2} \log \left(q^{-1} \psi(q)^{-1}\right)=\infty\end{cases}
$$

Evidently both these results are of Khintchine type, where the measure of a set of points $\psi$-approximable by a distance function is either null or full, according to the convergence or divergence of an 'area' series.

The above results suggest that a general Khintchine-type theorem might exist for star bodies. While Gallagher's result implies Khintchine type theorems for a number of star bodies, only convex star bodies and star bodies of the general shape as the ones in (3) are covered. The technical requirement for Gallagher's result (property $\mathrm{P}$ ) is that given a point $\left(p_{1}, p_{2}\right)$ inside the star body $S$, the entire rectangle with vertices $(0,0),\left(p_{1}, 0\right)$, $\left(p_{1}, p_{2}\right)$ and $\left(0, p_{2}\right)$ must be a subset of $S$.

It would also seem that a growth condition on the size of the unbounded parts of the star body would influence the breaking point (i.e., when the sum converges or diverges). In an earlier paper [9], the first author found a measure zero result of the above type under a technical covering condition. This work was subsequently generalised to systems of linear forms [10]. However, the specific star bodies to which these results can easily be applied are only minor generalisations of the examples above.

Some recent results in Diophantine approximation on manifolds due to Bernik, Kleinbock and Margulis [4, 14] suggest that there might be a connection between distance functions and Diophantine approximation and that a general Khintchine-type theorem might exist for general star bodies. In Section 2 below, we will prove Khintchine type theorems for a large class of distance functions. The results depend critically on the intrinsic arithmetic properties of the distance functions as well as their geometric properties. We shall state the results once we have the notational apparatus in place.

A tantalising result due to Aliev and Gruber [1] is in a certain sense dual to the problem studied in this paper and measures the set of lattices which have points inside a given star body of infinite volume. They show that given a star body $S \subseteq \mathbb{R}^{n}$ of infinite volume, almost all lattices have $n$ linearly independent primitive points inside $S$.

Associated with the sets $W\left(|\cdot|_{\infty} ; \psi\right)$ and $W\left(\left(x_{1}, x_{2}\right) \mapsto \sqrt{\left|x_{1}\right|\left|x_{2}\right|} ; \psi\right)$ are two transference principles, which relate the simultaneous Diophantine approximation of the coordinates of $x \in \mathbb{R}^{n}$ to the linear forms $q \cdot x$, where $x \in \mathbb{R}^{n}$ and $q \in \mathbb{Z}^{n}$. Let $\langle x\rangle=x-k_{x} \in$ $[1 / 2,1 / 2)^{n}$, where $k_{x} \in \mathbb{Z}^{n}$ denotes the symmetrised distance from $x \in \mathbb{R}^{n}$ to the nearest point in the integer lattice $\mathbb{Z}^{n}$. The following Transference Principle (see [18]; a different 
formulation is in [7, Chapter V, Theorem IV]) relates the properties of simultaneous Diophantine approximation, associated with the height, and those of the dual inner product form.

Khintchine's Transference Principle. Let $n \in \mathbb{N}$ and let $x \in(\mathbb{R} \backslash \mathbb{Q})^{n}$. The following two conditions are equivalent:

(i) For some $\varepsilon>0$, there are infinitely many $q \in \mathbb{Z}^{n}$ such that

$$
|\langle q \cdot x\rangle| \leq|q|^{-n-\varepsilon}
$$

(ii) For some $\varepsilon^{\prime}>0$, there are infinitely many $p \in \mathbb{Z}$ such that

$$
\|q x\| \leq|q|^{-\left(1+\varepsilon^{\prime}\right) / n} .
$$

Note that if condition (i) is true for some $\varepsilon>0$, it is automatically satisfied for all $\hat{\varepsilon}<\varepsilon$, and similarly for condition (ii).

There is an analogous multiplicative version for multiplicative Diophantine approximation and the inner product form. Sprindžuk [18, page 69] states it without proof (an $n$-th is root missing from the left hand side of his inequality (9)). Dyson [11] deduces it from a more general form of Khintchine's Transference Principle and [19 gives a contrapositive form using a method of A. Baker [2].

Theorem 1.1. Let $n \in \mathbb{N}$ and $x=\left(x_{1}, \ldots, x_{n}\right) \in(\mathbb{R} \backslash \mathbb{Q})^{n}$. The following two conditions are equivalent

(i) For some $\varepsilon>0$, there are infinitely many $q \in \mathbb{Z}^{n}$ such that

$$
|\langle q \cdot x\rangle| \leq\left(\prod_{i=1}^{n} \max \left(\left|q_{i}\right|, 1\right)\right)^{-1-\varepsilon} .
$$

(ii) For some $\varepsilon^{\prime}>0$, there are infinitely many $p \in \mathbb{Z}$ such that

$$
\left(\prod_{i=1}^{n}\left|\left\langle p x_{i}\right\rangle\right|\right)^{1 / n} \leq|p|^{-\left(1+\varepsilon^{\prime}\right) / n} .
$$

In Section 3, we shall give a direct proof of Theorem 1.1 which also gives a procedure for proving transference principles for other symmetric distance functions than $\left(x_{1}, \ldots, x_{n}\right) \mapsto\left(\prod\left|x_{i}\right|\right)^{1 / n}$. We will also outline an application to a distance function not covered by previous results. In this case, the assumption that the star bodies are symmetric about the origin is critical.

We will use the Vinogradov notation. That is, for two real numbers $x, y$, we will write $x \ll y$ if there exists a constant $C>0$ such that $x \leq C y$. If $x \ll y$ and $y \ll x$, we will write $x \asymp y$.

\section{KHINTCHINE TYPE THEOREMS}

We will treat two separate classes of distance functions, for which the theorems are of a different nature. We make the appropriate definitions and state the results before going on to prove the main theorems. In the final part of this section, we will discuss the remaining distance functions and conjectures about the corresponding Khintchine type theorems. 
2.1. Notation, definitions and statement of results. Let $F: \mathbb{R}^{2} \rightarrow[0, \infty)$ be a distance function. We define the skeleton of $F$ to be the set

$$
\operatorname{skel}(F)=F^{-1}(0) \text {. }
$$

It is easy to see, that the skeleton of a distance function consists of the origin together with a (possibly infinite and possibly zero) number of half-lines starting at the origin. For each such line, the star body has an unbounded component. If there are no such lines, the star body is bounded. Note that Gallagher's property P is only of interest when any half-lines in the skeleton lie on one of the coordinate axes.

Let $L$ be a half-line from the skeleton of $F$. We will call $L$ significant if the component around it carries an infinite amount of mass of the star body, i.e., if for any $M>0$,

$$
\left|\left\{x \in \mathbb{R}^{2}: F(x)<1\right\} \cap\left\{x \in \mathbb{R}^{2}: \operatorname{dist}(x, L)<M\right\}\right|=\infty .
$$

Note that the existence of a significant line in the skeleton of the star body immediately implies that the measure of the star body is infinite. Conversely, if the measure of an unbounded star body with only finitely many half-lines in its skeleton is infinite, then the skeleton contains a significant line.

When the skeleton of $F$ contains an irrationally sloped significant line, we prove the following result, generalising a result by the second author [15].

Theorem 2.1. Let $F: \mathbb{R}^{2} \rightarrow[0, \infty)$ be a distance function corresponding to an unbounded star body and let $\psi: \mathbb{N} \rightarrow(0, \infty)$ be some function. Suppose that that at least one of the (half-)lines in $\operatorname{skel}(F)$ is significant with an irrational slope. Suppose further that for each $\varepsilon>0$, the width $w(r)=w(r ; \varepsilon)>0$ of this unbounded component of $F^{-1}([0, \varepsilon))$ containing this line is non-increasing as the distance $r$ from the origin increases. Then, for almost all $x \in \mathbb{R}^{2}$ and any $q \in \mathbb{N}$, there are infinitely many $p \in \mathbb{Z}^{2}$ such that

$$
F(x-p)<\psi(q)
$$

whence

$$
|W(F ; \psi)|=1 \text {. }
$$

Thus the existence of an irrational significant line implies the stronger conclusion that for almost all $x \in \mathbb{R}^{2}$ and for any $\varepsilon$, there are infinitely many $p \in \mathbb{Z}^{2}$ for which $F(x-p)<\varepsilon$. Note that it is possible for a star body to be unbounded while having no significant lines in the skeleton. We will return to this case and others like it later. While the irrationality of the slope makes the result plausible, the result is not always true and a monotonicity condition is critical in the proof. The proof is not straightforward and uses recent work of Bugeaud on a variant of the usual form of Diophantine approximation 6 .

In the case when the skeleton of $F$ consists of finitely many half-lines whose slopes are rational numbers (or in the degenerate case when the skeleton is just the origin), we will need additional definitions. Let $F$ be such a distance function, let $n$ be the number of lines in the skeleton of $F$ and suppose that the slopes of these are $s_{i} / r_{i}$ for some integers $s_{i}, r_{i}, i=1, \ldots, n$. We see that if we take the union of the sets $\left\{x \in \mathbb{R}^{2}: F(x)<\rho\right\}+p$ where $p \in \mathbb{Z}^{2}$, we get a pattern which repeats itself with period $\hat{s}=\operatorname{lcm}\left(s_{1}, \ldots, s_{n}\right)$ in the direction of the first coordinate axis and with period $\hat{r}=\operatorname{lcm}\left(r_{1}, \ldots, r_{n}\right)$ in the direction of the second axis. We call such a rectangle $\mathcal{R}_{F}$ a fundamental rectangle for $F$. 
Let $\varepsilon>0$ and define the function $D_{F}$ of $\varepsilon$ by

$$
D_{F}(\varepsilon)=\frac{\left|\left(\bigcup_{p \in \mathbb{Z}^{2}}\left\{x \in \mathbb{R}^{2}: F(x)<\varepsilon\right\}+p\right) \cap \mathcal{R}_{F}\right|}{\left|\mathcal{R}_{F}\right|} .
$$

When the star body is bounded, the appropriate definition of the above quantity is

$$
D_{F}(\varepsilon)=\left|\left\{x \in \mathbb{R}^{2}: F(x)<\varepsilon\right\}\right| .
$$

We can now state our second Khintchine type theorem.

Theorem 2.2. Let $F: \mathbb{R}^{2} \rightarrow \mathbb{R}$ be a distance function such that $\operatorname{skel}(F)$ consists of finitely many lines, each with a rational slope. Let $\psi: \mathbb{N} \rightarrow(0, \infty)$ be a function such that $D_{F}(q \psi(q))$ is decreasing. Then

$$
|W(F ; \psi)|= \begin{cases}0 & \text { whenever } \sum_{q=1}^{\infty} D_{F}(q \psi(q))<\infty \\ 1 & \text { whenever } \sum_{q=1}^{\infty} D_{F}(q \psi(q))=\infty .\end{cases}
$$

Note that Theorem 2.2 covers (2), (3) and a number of additional cases, including all distance functions corresponding to bounded star bodies as well as all the cases covered by Gallagher's result [12. Note also, that $\left|\mathcal{R}_{F}\right|$ depends only on the distance function, and so the series in the statement of Theorem 2.2 could be replaced by volume sums, as is the case in [12].

2.2. The irrational case. In this section, we prove Theorem 2.1. Let $q \in \mathbb{N}$ be fixed. Let

$$
L=\{(x, \alpha x): x \in[0, \infty)\}
$$

denote a line through the origin in $\operatorname{skel}(F)$ with irrational slope $\alpha=\tan \theta$. Suppose that $\alpha>1$, as the other case may be treated analogously by interchanging the axes. Identify each $L$ with its canonical projection into the unit square, which is in turn identified with the two-torus $\mathbb{T}^{2}$. Since $L$ has irrational slope, it follows from Kronecker's Theorem (see e.g. [13, Proposition 1.5.1]) that each of these geodesics is dense in $\mathbb{T}^{2}$. However, this is not sufficient to imply Theorem 2.1 for significant lines.

Consider a fixed but arbitrary horizontal line $H=\left\{\left(x, y_{0}\right): 0 \leq x<1\right\}$, where $0 \leq y_{0}<$ 1 , through the unit square $\mathbb{T}^{2} ; H$ will be identified with the circle $\mathbb{T}^{1}$ (one dimensional torus). Consider the geodesic

$$
L=\left\{\left(\frac{y}{\alpha}, y\right): y \in[0, \infty)\right\} .
$$

The point $\left(\left(y_{0}+n\right) / \alpha, y_{0}+n\right)=\left(y_{0} / \alpha, y_{0}\right)+n(1 / \alpha, 1)$ on $L$ is at distance

$$
\left.r_{n}=\left(\left(y_{0}+n\right) / \alpha\right)^{2}+\left(y_{0}+n\right)^{2}\right)^{1 / 2}=\left(y_{0}+n\right) \operatorname{cosec} \theta
$$

from the origin and projects to the point

$$
\left(\left\{\frac{y_{0}+n}{\alpha}\right\},\left\{y_{0}+n\right\}\right)=\left(\left\{\frac{y_{0}+n}{\alpha}\right\}, y_{0}\right) \in \mathbb{T}^{2} .
$$

As the slope of $L$ is irrational, the geodesic intersects the horizontal line $H$ first at the point $\left(x_{0}, y_{0}\right)$, where since $\alpha>1$,

$$
x_{0}=x_{0}(\alpha)=\frac{y_{0}}{\alpha}<1
$$


and thereafter at the distinct points $\left(T^{n} x_{0}, y_{0}\right)$, where $n=1,2, \ldots$ and the map $T: \mathbb{T}^{1} \rightarrow$ $\mathbb{T}^{1}$ can be regarded as a rotation of the circle by the irrational angle $\alpha$ and

$$
T^{n} x_{0}:=x_{n}=\left\{x_{0}+n \alpha^{-1}\right\} .
$$

Let $L_{n}$ be the directed segment which meets

$$
\left(T^{n} x_{0}, y_{0}\right)=\left(x_{n}, y_{0}\right)=\left(\left\{x_{0}+n \alpha^{-1}\right\}, y_{0}\right)
$$

from below. The distance $r_{n}$ along the irrational line segments $L_{0}, L_{1}, \ldots$ from the origin to the point $\left(x_{n}, y_{0}\right)=\left(\left\{x_{0}+n \alpha^{-1}\right\}, y_{0}\right)$ is given by (10).

Suppose $L$ a significant line. Assume for the sake of simplicity that the star body is approximately symmetric about significant lines, that is, for any point $(x, y)$ on a significant line $L$, the distance from $(x, y)$ to the upper part of the boundary $F^{-1}(\psi(q))$ is comparable to the distance from the lower part. More precisely, denote by $w^{ \pm}=$ $w^{ \pm}(r, \psi(q))$ the perpendicular distances of the point $(x, y) \in L$, where $r^{2}=x^{2}+y^{2}$, from the upper $(+)$ and lower $(-)$ boundaries $\partial F^{-1}([0, \psi(q)))$ of the starbody, so that the width $w=w(r, \psi(q))$ of the $\psi(q)$-neighbourhood at $\left(x_{n}, y_{0}\right)$ is given by $w=w^{+}+w^{-}$. Writing $w_{n}^{+}=w^{+}\left(\left(y_{0}+n\right) \operatorname{cosec} \theta, \psi(q)\right)$ and similarly for $w_{n}^{-}$and $w_{n}$, we have

$$
w_{n}=w_{n}^{+}+w_{n}^{-},
$$

where $w_{n}^{+} \asymp w_{n}^{-} \asymp w_{n}$.

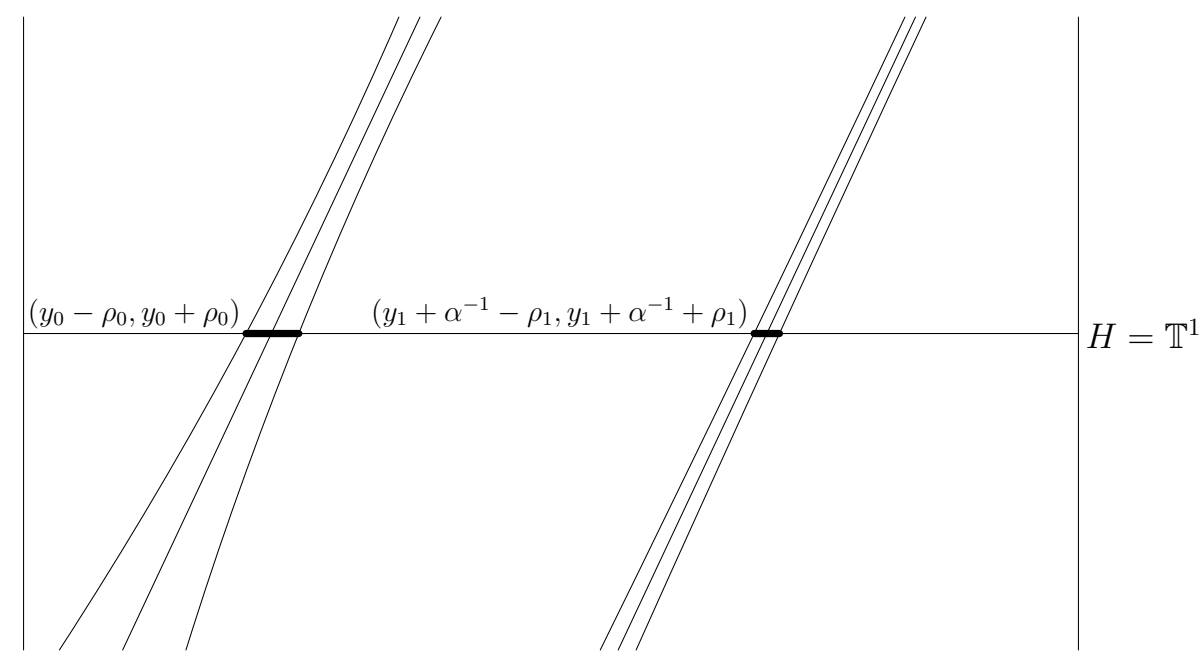

FiguRE 1. Two consecutive intervals $I_{n}$ and $I_{n+1}$

Let $I_{n}$ be the interval formed by the intersection of $H$ and the $\psi(q)$-neighbourhood of the segment $L_{n}$ and its continuation through $\left(x_{n}, y_{0}\right)$ (see Figure 1). Then the length $\left|I_{n}\right|$ of the interval $I_{n}$ satisfies

$$
w_{n} \operatorname{cosec} \theta \ll\left|I_{n}\right| \ll w_{n} \operatorname{cosec} \theta,
$$

that is, $w_{n} \asymp\left|I_{n}\right|$. Inscribe an interval centred at $\left(x_{n}, y_{0}\right)$ with radius

$$
\sigma_{n}=K \min \left\{w_{n}^{+}, w_{n}^{-}\right\} .
$$

where $K$ is a sufficiently small positive constant chosen so that

$$
\tilde{I}_{n}:=\left\{x \in \mathbb{T}^{1}:\left\|x-\left\{x_{0}-n \alpha^{-1}\right\}\right\|<\sigma_{n}\right\} \subseteq I_{n},
$$


where $\|x\|$ denotes the distance to nearest integer (this is possible by the assumption of approximate symmetry of the star body). Then $\sigma_{n} \asymp w_{n}$ and $\left|\tilde{I}_{n}\right| \asymp\left|I_{n}\right|$. We show that given $q \in \mathbb{N}$, almost all $\left(x, y_{0}\right) \in H$ lie in the interval $\tilde{I}_{n} \subseteq I_{n}$ for infinitely many $n$.

Consider the 'limsup' set $E\left(y_{0}\right)$ of points $x$ such that the point $\left(x, y_{0}\right)$ falls into infinitely many of the intervals $\tilde{I}_{n}$ in $H$. Now

$$
\sum_{n}\left|\tilde{I}_{n}\right| \asymp \sum_{n}\left|I_{n}\right|
$$

and

$$
\sum_{n}\left|I_{n}\right| \asymp \sum_{n} \sigma_{n} \asymp \sum_{n} w\left(r_{n}, \psi(q)\right) \asymp \sum_{n} w((y+n) \operatorname{cosec} \theta, \psi(q)) .
$$

But

$$
\sum_{n}\left|I_{n}\right| \asymp \int w(u \operatorname{cosec} \theta, \psi(q)) d u \asymp \int w(v, \psi(q)) d v=\infty,
$$

since by assumption $w(u, \psi(q))$ is non-increasing and since the line $L$ is significant. Thus

$$
\sum_{n}\left|\tilde{I}_{n}\right|=\infty
$$

We now show that $E\left(y_{0}\right)$ has full measure. By 3 it suffices to prove local ubiquity of the points $x_{0}+\{n / \alpha\}, n=1,2, \ldots$ relative to an appropriate function $\lambda$. This relies on the so-called Three Distances Theorem due to Sós [17] and to a certain extent follows the methods used by Bugeaud in [6] (see also [16] for an approach using continued fractions).

Let $\lambda:(0, \infty) \rightarrow(0, \infty)$ be a function decreasing to zero and let $\left(N_{r}\right)$ be a strictly increasing sequence of natural numbers. The set of points $\mathcal{R}=\left\{z_{n}: n \in \mathbb{N}\right\}$ in the metric space $\mathbb{T}^{1}$ is said to be locally ubiquitous relative to $\lambda$ and $\left(N_{r}\right)$ if there is a constant $\kappa>0$ such that for any interval $I=(c-\rho, c+\rho)$ with $\rho$ sufficiently small and any $r \geq r_{0}(I)$,

$$
\mu\left(I \cap \bigcup_{N_{r} \leq n<N_{r+1}}\left(z_{n}-\lambda\left(N_{r}\right), z_{n}+\lambda\left(N_{r}\right)\right)\right) \geq 2 \kappa \rho .
$$

This quantitative version of the local density of the points in $\mathcal{R}$ is the appropriate specialisation of the definition of local ubiquity in $[3]$.

Lemma 2.3. Let $\mathcal{R}=\left\{\left\{x_{0}+n \alpha^{-1}\right\}: n \in \mathbb{N}\right\}$. The system $(\mathcal{R}, n)$ is locally ubiquitous relative to the function $\lambda(N)=3 /\left(N_{r}+1\right)$, where $N_{r} \leq N<N_{r+1}$ for an appropriately chosen strictly increasing sequence of natural numbers $\left(N_{r}\right)$.

Proof. As in the proof of [․․ Proposition 1], we find that by the Three Distances Theorem, for any $N \in \mathbb{N}$, the sequence of points

$$
\left\{x_{0}+\alpha^{-1}\right\},\left\{x_{0}+2 \alpha^{-1}\right\}, \ldots,\left\{x_{0}+N \alpha^{-1}\right\}
$$

partition the circle $\mathbb{T}^{1}$ into $N+1$ intervals with lengths from a set of cardinality at most three, where $\{x\}$ denotes the fractional part of $x$. The largest of these distances may be seen to be less than or equal to $3 /(N+1)$ for infinitely many positive integers $N$. Call this sequence $\left(N_{r}\right)$. Hence, for any $N \in\left[N_{r}, N_{r+1}\right]$, the intervals centred at the points $\left\{\alpha^{-1}\right\}, \ldots,\left\{N \alpha^{-1}\right\}$ of length $3 /\left(N_{r}+1\right)$ cover the circle. Therefore the system is locally ubiquitous relative to $\lambda$ and $\left(N_{r}\right)$. 
Consider the family of intervals $\tilde{I}_{n}$. The centres of these intervals form a ubiquitous system relative to $\lambda$ and it is an easy consequence of [3, Theorem 2] that the divergence of the sum $\sum_{n}\left|\tilde{I}_{n}\right|$ implies that the limsup set $E\left(y_{0}\right)$ has full measure. It follows that for any fixed $y_{0} \in[0,1)$, for almost there exist infinitely many $p \in \mathbb{Z}^{2}$ such that

$$
F\left(\left(x, y_{0}\right)-p\right)<\psi(q) \text {. }
$$

Let $\chi_{E(y)}$ denote the characteristic function of $E(y)$. Theorem 2.1 now follows from Fubini's theorem, since

$$
\iint_{[0,1)^{2}} \chi_{E(y)}(x, y) d x d y=\int_{0}^{1} \int_{0}^{1} \chi_{E(y)}(x, y) d x d y=\int_{0}^{1} 1 d y=1,
$$

whence a fortiori, for any $q \in \mathbb{N}$,

$$
\mid\left\{(x, y) \in \mathbb{T}^{2}: F((x, y)-p)<\psi(q) \text { for infinitely many } p \in \mathbb{Z}^{2}\right\} \mid=1 .
$$

In the more general case, i.e., when the star body is not assumed to be approximately symmetric about significant lines, the above argument can be extended by changing the resonant points in the ubiquitous system to the centres of the intervals $I_{n}$. These do not have to be as regularly spaced as the resonant points considered in the special case, but this difficulty can be resolved by adding an additional term to the ubiquity function $\lambda$ in Lemma 2.3. In this case, the ubiquity function should be

$$
\lambda(N)=3 /\left(N_{r}+1\right)+\max \left\{w_{N_{r}}^{+}, w_{N_{r}}^{-}\right\} .
$$

If necessary, we may take a smaller star body inside the original one for which $\max \left\{w_{N_{r}}^{+}, w_{N_{r}}^{-}\right\}$ is not dominating the main term, but for which the corresponding line remains significant. The details are left to the interested reader.

Note that if the unbounded starbody has no significant lines (so that $F^{-1}([0, \varepsilon)$ ) has finite volume), then given $q \in \mathbb{N}$, the set of points $(x, y) \in \mathbb{T}^{2}$ for which

$$
F((x, y)-p)<\psi(q)
$$

for infinitely many $p \in \mathbb{Z}^{2}$ is null by the Borel-Cantelli lemma. The question of the measure of the set of $(x, y)$ for which

$$
F\left((x, y)-\frac{p}{q}\right)<\psi(q)
$$

holds for infinitely many $p / q$ is open.

2.3. The rational case. For completeness, we first deal with the degenerate case when the skeleton consists solely of the origin, corresponding to $F$ being a gauge function [8].

Lemma 2.4. Let $S \subseteq \mathbb{R}^{2}$ be a bounded star body with corresponding distance function F. Then

$$
|W(F ; \psi)|= \begin{cases}0 & \text { if } \sum_{q=1}^{\infty} D_{F}(q \psi(q))<\infty \\ 1 & \text { if } \sum_{q=1}^{\infty} D_{F}(q \psi(q))=\infty\end{cases}
$$


Proof. By the final corollary of [8, Section IV.2], there are constants $c, C>0$ such that for any $x \in \mathbb{R}^{2}$,

$$
c|x|_{\infty} \leq F(x) \leq C|x|_{\infty},
$$

whence

$$
W\left(|\cdot|_{\infty} ; \frac{\psi}{C}\right) \subseteq W(F ; \psi) \subseteq W\left(|\cdot|_{\infty} ; \frac{\psi}{c}\right)
$$

Applying (2) immediately gives the result.

We now suppose that the skeleton of the star body contains a half-line and prove the two cases of Theorem 2.2 separately. First, for any $q \in \mathbb{N}$ and any $\varepsilon>0$, we define the resonant neighbourhood

$$
B_{q}(F, \varepsilon)=\bigcup_{p \in \mathbb{Z}^{2}}\left\{x \in \mathbb{T}^{2}: F(x-p / q)<\varepsilon\right\} .
$$

The measure of the resonant neighbourhood is closely related to $D_{F}(\varepsilon)$. Note first that

$$
\left|\frac{1}{q} \mathcal{R}_{F} \cap B_{q}(F, \varepsilon)\right|=\frac{\hat{s} \hat{r}}{q^{2}} D_{F}(q \varepsilon),
$$

as is seen by scaling the set to be estimated by $q$ and using homogeneity of the distance function.

To obtain the measure of $B_{q}(F, \varepsilon)$, we tile $\mathbb{R}^{2}$ by disjoint translates of $\frac{1}{q} \mathcal{R}_{F}$. Inside each of these disjoint sets, we find a translated copy of $\frac{1}{q} \mathcal{R}_{F} \cap B_{q}(F, \varepsilon)$, and the union of these sets cover $B_{q}(F, \varepsilon)$. Hence, to estimate the measure of $B_{q}(F, \varepsilon)$, it suffices to count the maximal (resp. minimal) number of disjoint translates of $\frac{1}{q} \mathcal{R}_{F}$ that can fit inside (resp. are needed to cover) the unit square, and multiply the result by the estimate from (15). In this way, we get

$$
\left[\frac{q}{\hat{r}}\right]\left[\frac{q}{\hat{s}}\right] \frac{\hat{s} \hat{r}}{q^{2}} D_{F}(q \varepsilon) \leq\left|B_{q}(F, \varepsilon)\right| \leq\left(\frac{q}{\hat{s}}+1\right)\left(\frac{q}{\hat{r}}+1\right) \frac{\hat{s} \hat{r}}{q^{2}} D_{F}(q \varepsilon)
$$

whence

$$
\left|B_{q}(F, \varepsilon)\right| \asymp D_{F}(q \varepsilon) .
$$

Applying the Borel-Cantelli Lemma [5], Lemma 3.14] to the resonant neighbourhoods with the measure estimate (16) yields a condition for $|W(F ; \psi)|$ to be zero. Indeed, whenever

$$
\sum_{q=1}^{\infty} D_{F}(q \psi(q))<\infty
$$

$W(F ; \psi)$ must be null. We have shown:

Lemma 2.5. Let $\psi: \mathbb{Z} \rightarrow(0, \infty)$ be some decreasing function and let $F: \mathbb{R}^{2} \rightarrow \mathbb{R}$ be a distance function such that the lines in $\operatorname{skel}(F)$ all have rational slopes. Let $D_{F}(\varepsilon)$ be defined as above. Suppose that

$$
\sum_{q=1}^{\infty} D_{F}(q \psi(q))<\infty .
$$

Then $|W(F ; \psi)|=0$. 
We now aim to obtain the corresponding divergence result, i.e., when the series (17) diverges, we expect the measure of $W(F ; \psi)$ to be full. Suppose first that the central part of the star body carries the bulk of the mass, i.e., there is a distance function $\widetilde{F}$ such that

(i) $\widetilde{F}$ determines a bounded star body.

(ii) $F(x) \leq \widetilde{F}(x)$ for all $x$ (or equivalently, the star body defined by $\widetilde{F}$ is a subset of the one defined by $F$ by [8. Corollary, p. 107]).

(iii) $D_{\widetilde{F}}(\varepsilon) \asymp D_{F}(\varepsilon)$ for $\varepsilon>0$ small enough.

In this case, $W(\widetilde{F} ; \psi) \subseteq W(F ; \psi)$, so the conclusion of Theorem 2.2 is ensured by Lemma 2.4. We have shown

Lemma 2.6. Let $F$ be a distance function and suppose that $\operatorname{skel}(F)$ consists of lines with rational slopes. Suppose further that there is a distance function $\widetilde{F}$ satisfying (i)-(iii) above. Then Theorem 2.2 holds for this distance function.

Suppose now that the central part of the star body does not carry the bulk of the mass, so that there is a cusp which includes an infinite amount of the mass. We will construct a subset of $W(F ; \psi)$, which is better behaved but which still has full measure. In brief, we will truncate the star body in such a way that only the unbounded component which carries most of the mass will remain. Subsequently, we will take the limsup set of these truncated sets, for which we can calculate the measure. For convenience we denote by $F_{\varepsilon}^{*}$ the distance function associated with the star body $\{\operatorname{dist}(x, L)<w(\varepsilon)\} \cap\{F(x)<\varepsilon\}$.

Lemma 2.7. Let $F$ be a distance function as in the statement of Theorem 2.2. Then there exists a function $w:(0, \infty) \rightarrow(0, \infty)$ with $w(\varepsilon)$ tending to zero as $\varepsilon$ tends to zero together with a (half-)line $L \in \operatorname{skel}(F)$ such that

$$
D_{F_{\varepsilon}^{*}}(1) \asymp D_{F}(\varepsilon),
$$

and so that the intersection between any line perpendicular to $L$ and the star body associated to $F_{\varepsilon}^{*}$ is a line segment.

Remark: We have to choose an entire family of distance functions rather than just a single one. This is to preserve the scaling properties of the original star body. This takes us away from the problem of approximation with respect to distance functions, but as we shall see, only the shape of the sets studied are of importance for the remainder of the proof.

Proof. We choose the line in $\operatorname{skel}(F)$ about which a significant proportion of the mass is concentrated. Such a line must exist, since there are only finitely many lines in skel $(F)$. By truncating the star body about this line, it is now straightforward to choose the function $w$ in such a way that (18) holds. To ensure that the second property holds, we choose $w$ as small as possible. For at least one of the lines in the $\operatorname{skel}(F)$, we will have both properties satisfied. Indeed, otherwise most of the mass would be concentrated in the cental part of the star body associated to $F$.

Lemma 2.8. Let $F_{1}^{\prime}, F_{2}^{\prime}: \mathbb{R}^{2} \rightarrow \mathbb{R}$ be distance functions with $\operatorname{skel}\left(F_{1}^{\prime}\right)=\operatorname{skel}\left(F_{2}^{\prime}\right)=L$, a (half-) line. Let $\delta_{1}, \delta_{2}>0$. Let $v \in \mathbb{R}^{2}$ be a unit vector in the direction of $L$ and let $v^{\perp}$ be 
a unit normal to $L$. Let $t_{1}, t_{2} \in \mathbb{R}$. The measure

$$
\left|\left\{x \in \mathcal{R}_{F_{1}^{\prime}}: F_{1}^{\prime}(x)<\delta_{1}\right\} \cap\left\{x \in \mathcal{R}_{F_{2}^{\prime}}: F_{2}^{\prime}\left(x+t_{1} v+t_{2} v^{\perp}\right)<\delta_{2}\right\}\right|
$$

decreases as $\left|t_{i}\right|$ increases and the other coordinate is fixed.

Proof. We define the characteristic functions $\chi_{1}$ and $\chi_{2}$ of $\left\{x \in \mathcal{R}_{F_{1}^{\prime}}: F_{1}^{\prime}(x)<\delta_{1}\right\}$ and $\left\{x \in \mathcal{R}_{F_{2}^{\prime}}: F_{2}^{\prime}(x)<\delta_{2}\right\}$ respectively. Clearly, for fixed $t_{1}$, the function $\chi_{1}(x) \chi_{2}\left(x+t_{1} v+\right.$ $\left.t_{2} v^{\perp}\right)$ is the characteristic function of an interval in the $t_{2}$ coordinate, and so decreasing in $\left|t_{2}\right|$ and analogously for fixed $t_{2}$. Hence,

$$
\begin{aligned}
\mid\left\{x \in \mathcal{R}_{F_{1}^{\prime}}: F_{1}^{\prime}(x)<\delta_{1}\right\} \cap\left\{x \in \mathcal{R}_{F_{2}^{\prime}}: F_{2}^{\prime}\left(x+t_{1} v+\right.\right. & \left.\left.t_{2} v^{\perp}\right)<\delta_{2}\right\} \mid \\
& =\int_{\mathbb{R}^{2}} \chi_{1}(x) \chi_{2}\left(x+t_{1} v+t_{2} v^{\perp}\right) d x
\end{aligned}
$$

also decreases with $\left|t_{i}\right|$ when the other coordinate is fixed.

We will now estimate the overlap between two resonant neighbourhoods in terms of the measure of the star bodies defined in Lemma 2.7. To ease notation, let $B_{q}^{*}$ be defined as $B_{q}(F, \psi(q))$ in (14) under the additional restriction that $\left(p_{1} \hat{r}, q\right)=\left(p_{2} \hat{s}, q\right)=1$. Furthermore, let

$$
A_{q}^{*}=\left\{x \in \mathbb{T}^{2}: F_{q \psi(q)}^{*}(x)<1\right\},
$$

where $F_{q \psi(q)}^{*}$ is the distance function from Lemma 2.7 ,

Lemma 2.9. Let $q, q^{\prime} \in \mathbb{N}$. Then

$$
\left|B_{q}^{*} \cap B_{q^{\prime}}^{*}\right| \leq\left|A_{q}^{*}\right|\left|A_{q^{\prime}}^{*}\right| \asymp D_{F}(q \psi(q)) D_{F}\left(q^{\prime} \psi\left(q^{\prime}\right)\right) .
$$

Proof. The last asymptotic equality follows at once from Lemma 2.7. We concentrate on the first inequality. First, we note that

$$
B_{q}^{*} \subseteq \bigcup \frac{A_{q}+\left(\begin{array}{c}
k_{1} \hat{r} \\
k_{2} \hat{s}
\end{array}\right)}{q},
$$

where the union is taken over all integers $k_{1}, k_{2}$ with $0 \leq k_{1} \hat{r} \leq q$ and $0 \leq l_{1} \hat{r}$ such that $\left(q, k_{1} \hat{r}\right)=\left(q, k_{2} \hat{s}\right)=1$. Hence

$$
B_{q}^{*} \cap B_{q^{\prime}}^{*} \subseteq \bigcup\left(\frac{A_{q}+\left(\begin{array}{c}
k_{1} \hat{r} \\
k_{2} \hat{s}
\end{array}\right)}{q} \cap \frac{A_{q^{\prime}}+\left(\begin{array}{c}
l_{1} \hat{r} \\
l_{2} \hat{s}
\end{array}\right)}{q^{\prime}}\right),
$$

where the union is taken over all integers $k_{1}, k_{2}, l_{1}, l_{2}$ with $0 \leq k_{1} \hat{r}, k_{2} \hat{s} \leq q$ and $0 \leq$ $l_{1} \hat{r}, l_{2} \hat{s} \leq q^{\prime}$ such that $\left(q, k_{1} \hat{r}\right)=\left(q, k_{2} \hat{s}\right)=\left(q^{\prime}, l_{1} \hat{r}\right)=\left(q^{\prime}, l_{2} \hat{s}\right)=1$. Consequently,

$$
\begin{aligned}
\left|B_{q}^{*} \cap B_{q^{\prime}}^{*}\right| & \leq \sum\left|\frac{A_{q}+\left(\begin{array}{c}
k_{1} \hat{r} \\
k_{2} \hat{s}
\end{array}\right)}{q} \cap \frac{A_{q^{\prime}}+\left(\begin{array}{c}
l_{1} \hat{r} \\
l_{2} \hat{s}
\end{array}\right)}{q^{\prime}}\right| \\
& =\sum\left|\frac{A_{q}}{q} \cap\left(\frac{A_{q^{\prime}}}{q^{\prime}}+\frac{1}{q q^{\prime}}\left(\begin{array}{c}
\left(l_{1} q-k_{1} q^{\prime}\right) \hat{r} \\
\left(l_{2} q-k_{2} q^{\prime}\right) \hat{s}
\end{array}\right)\right)\right|,
\end{aligned}
$$

where the summation range is as above. Under the summation conditions, there are at most $\left(q, q^{\prime}\right)^{2}$ repeated summands in the sums in (19). Hence, on changing the summation 
ranges, we get

$$
\left|B_{q}^{*} \cap B_{q^{\prime}}^{*}\right| \leq \sum_{\left(j_{1}, j_{2}\right) \in \mathbb{Z}^{2}}\left|\frac{A_{q}}{q} \cap\left(\frac{A_{q^{\prime}}}{q^{\prime}}+\frac{\left(q, q^{\prime}\right)}{q q^{\prime}}\left(\begin{array}{c}
j_{1} \hat{r} \\
j_{2} \hat{s}
\end{array}\right)\right)\right| .
$$

Consider now the function

$$
f\left(t_{1}, t_{2}\right)=\left|\frac{A_{q}}{q} \cap\left(\frac{A_{q^{\prime}}}{q^{\prime}}+\frac{\left(q, q^{\prime}\right)}{q q^{\prime}}\left(\begin{array}{c}
t_{1} \hat{r} \\
t_{2} \hat{s}
\end{array}\right)\right)\right| .
$$

As a consequence of Lemma 2.8, this function decreases as $\left|t_{i}\right|$ increases $(i=1,2)$. Conseqently,

$$
\int_{0}^{1} \int_{0}^{1} f\left(t_{1}, t_{2}\right) d t_{1} d t_{2} \geq f(1,1) .
$$

Using this and translation invariance of Lebesgue measure in (20), we obtain

$$
\begin{aligned}
\left|B_{q}^{*} \cap B_{q^{\prime}}^{*}\right| & \leq\left(q, q^{\prime}\right)^{2} \sum_{\left(j_{1}, j_{2}\right) \in \mathbb{Z}^{2}} \iint_{[0,1)^{2}+\left(j_{1}, j_{2}\right)} f\left(t_{1}, t_{2}\right) d t_{1} d t_{2} \\
& =\left(q, q^{\prime}\right)^{2} \iint_{\mathbb{R}^{2}}\left|\frac{A_{q}}{q} \cap\left(\frac{A_{q^{\prime}}}{q^{\prime}}+\frac{\left(q, q^{\prime}\right)}{q q^{\prime}}\left(\begin{array}{c}
t_{1} \hat{r} \\
t_{2} \hat{s}
\end{array}\right)\right)\right| d t_{1} d t_{2} \\
& =\iint_{A_{q}}\left|A_{q^{\prime}}\right| d t_{1} d t_{2} \\
& =\left|A_{q}\right|\left|A_{q^{\prime}}\right| .
\end{aligned}
$$

This completes the proof.

The following estimate uses elementary number theory and summation by parts (see [7. Chapter VII, Lemma 7]).

Lemma 2.10. Let $\omega(q)$ be monotonely decreasing and positive. Let $\phi(q)$ denote the Euler $\phi$-function of $q$. Then

$$
\sum_{q=1}^{N}\left(\frac{\phi(q)}{q}\right)^{2} \omega(q) \gg \sum_{q=2}^{N} \omega(q) .
$$

The above lemma will be useful because of the following:

Lemma 2.11. Suppose that $q \in \mathbb{N}$ satisfies $(q, \hat{r})=(q, \hat{s})=1$. Then,

$$
\left|B_{q}^{*}\right| \gg\left(\frac{\phi(q)}{q}\right)^{2} D_{F}(q \psi(q)) .
$$

Proof. This follows from the definition of $B_{q}^{*}$. Indeed, the number of sets under the union in the definition is $\asymp(\phi(q) / q)^{2}$, as is easily seen by counting the number of $\left(p_{1}, p_{2}\right)$ satisfying the co-primality condition. The co-primality condition and the fact that $D_{F}(q \psi(q))$ is non-increasing implies that the disjoint components are dominant.

Note that Lemma 2.10 and Lemma 2.11 together with the divergence assumption imply that

$$
\sum_{\substack{q \geq 1 \\(q, \hat{r})=(q, \hat{s})=1}}\left|B_{q}^{*}\right|=\infty
$$


Indeed, the set of $q \in \mathbb{N}$ with $(q, \hat{r})=(q, \hat{s})=1$ contains the arithmetic progression $\{\hat{r} \hat{s} n+1\}_{n=0}^{\infty}$, and so has positive density. On noting that the terms of the series are decreasing over the full range of $q$, (21) follows by a simple change of variables.

Lemma 2.12. Let $F: \mathbb{R}^{2} \rightarrow[0, \infty)$ be a distance function such that the lines in $\operatorname{skel}(F)$ have rational slopes. Let $\psi: \mathbb{N} \rightarrow[0, \infty)$ be a function such that $D_{F}(q \psi(q))$ is nonincreasing and suppose that

$$
\sum_{q=1}^{\infty} D_{F}(q \psi(q))=\infty
$$

Then $|W(F ; \psi)|=1$.

Proof. Denote by $\sum_{q, q^{\prime}}^{N}$ the sum over all $q, q^{\prime} \in \mathbb{Z}$ such that $1 \leq q, q^{\prime} \leq N,(q, \hat{r})=(q, \hat{s})=$ $1,\left(q^{\prime}, \hat{r}\right)=\left(q^{\prime}, \hat{s}\right)=1$, and similarly by $\sum_{q}^{N}$ the sum over all $q \in \mathbb{Z}$ such that $1 \leq q \leq N$, $(q, \hat{r})=(q, \hat{s})=1$. Applying a quasi-independent divergence case of the Borel-Cantelli Lemma (see Lemma 5 in [18]), we see that

$$
\begin{aligned}
|W(F ; \psi)| & \geq \limsup _{N \rightarrow \infty} \frac{\left(\sum_{q}^{N}\left|B_{q}^{*}\right|\right)^{2}}{\sum_{q, q^{\prime}}^{N}\left|B_{q}^{*} \cap B_{q^{\prime}}^{*}\right|} \\
& \gg \limsup _{N \rightarrow \infty} \frac{\left(\sum_{q}^{N}\left(\frac{\phi(q)}{q}\right)^{2}\left|B_{q}(F, \psi(q))\right|\right)^{2}}{\sum_{q, q^{\prime}}^{N}\left|A_{q}\right|\left|A_{q^{\prime}}\right|} \\
& \gg \limsup _{N \rightarrow \infty} \frac{\left(\sum_{q}^{N} D_{F}(q \psi(q))\right)^{2}}{\left(\sum_{q}^{N} D_{F}(q \psi(q))\right)^{2}} \gg 1
\end{aligned}
$$

by Lemmas 2.9, 2.11 and 2.10. As $W^{*}(F ; \psi) \subseteq W(F ; \psi)$, we clearly have $|W(F ; \psi)| \geq$ $c>0$ for some $c>0$.

We now apply an 'inflation' argument taken from [7, Chapter VII]. Let $\eta: \mathbb{N} \rightarrow(0,1]$ be a function which decreases to zero so slowly that $\sum_{q} D_{F}(q \eta(q) \psi(q))=\infty$. Such a function can be found as follows. First, define a strictly inreasing sequence $q_{j}$ such that $\sum_{q_{j} \leq q<q_{j+1}} D_{F}(q \psi(q))>1$ for any $j \in \mathbb{N}$. Subsequently, define a sequence of positive real numbers $\eta_{j}$ by the equation

$$
D_{F}\left(\eta_{j} q_{j+1} \psi\left(q_{j+1}\right)\right)=\frac{1}{j} D_{F}\left(q_{j+1} \psi\left(q_{j+1}\right)\right)
$$

Such numbers exist by continuity of the distance function and since $q \psi(q)$ is non-increasing. Finally, let

$$
\eta(q)=\eta_{j}, q_{j} \leq q<q_{j+1} .
$$


In is now immediate that

$$
\begin{aligned}
\sum_{q=1}^{\infty} D_{F}(q \eta(q) \psi(q)) & =\sum_{j=1}^{\infty} \sum_{q_{j} \leq q<q_{j+1}} D_{F}(q \eta(q) \psi(q)) \\
& \geq \sum_{j=1}^{\infty} \sum_{q_{j} \leq q<q_{j+1}} D_{F}\left(\eta_{j} q_{j+1} \psi\left(q_{j+1}\right)\right)>\sum_{j=1}^{\infty} \frac{1}{j}=\infty .
\end{aligned}
$$

Let the function $\psi_{\eta}: \mathbb{N} \rightarrow[0, \infty)$ be defined by $\psi_{\eta}(q)=\eta(q) \psi(q)$. Going through the above argument with $\psi_{\eta}$ in place of $\psi$, we easily find that $\left|W\left(F ; \psi_{\eta}\right)\right|>c>0$. Let $x_{0} \in W\left(F ; \psi_{\eta}\right)$ be a point of metric density for $W\left(F ; \psi_{\eta}\right)$ and let $\varepsilon \in(0,1)$ be arbitrary. By Lebesgue's Density Theorem, there is an $n_{0} \in \mathbb{N}$ and a box $H$ centred at $x_{0}$ of measure $|H|=1 / n_{0}$ such that

$$
\frac{\left|W\left(F ; \psi_{\eta}\right) \cap H\right|}{|H|}=n_{0}\left|W\left(F ; \psi_{\eta}\right) \cap H\right| \geq 1-\varepsilon .
$$

Thus $\left|n_{0}\left(W\left(F ; \psi_{\eta}\right) \cap H\right)\right| \geq 1-\varepsilon$, so there is a set $U \in \mathbb{T}^{2}$ of measure at least $1-\varepsilon$ such that every point $x \in U$ is of the form $x=n_{0} x^{\prime}+p, p \in \mathbb{Z}^{2}, x^{\prime} \in W\left(F ; \psi_{\eta}\right)$. We claim that $U \subseteq W\left(F ; n_{0} \psi_{\eta}\right)$. Indeed, let $x \in U$. Then

$$
F\left(x-\frac{p^{\prime}}{q}\right)=F\left(n_{0} x^{\prime}+p-\frac{p^{\prime}}{q}\right)=n_{0} F\left(x^{\prime}-\frac{q p-p^{\prime}}{n_{0} q}\right) .
$$

As $p^{\prime} \in \mathbb{Z}^{2}$ varies freely, ratio $\left(p^{\prime}-p q\right) / n_{0} q$ varies freely over the lattice $1 / n_{0} q \mathbb{Z}^{2}$ which contains $1 / q \mathbb{Z}^{2}$. Hence, as $x^{\prime} \in W\left(F ; \psi_{\eta}\right)$, for infinitely many $q \in \mathbb{Z}$ there is a $p^{\prime} \in \mathbb{Z}^{2}$ such that

$$
F\left(x-p^{\prime} / q\right)<n_{0} \eta(q) \psi(q) .
$$

But there exists $Q$ such that $q \geq Q$ implies that $\eta(q) \leq n_{0}^{-1}$, whence

$$
F\left(x-p^{\prime} / q\right)<\psi(q)
$$

for infinitely many $q$ and so $x \in W(F ; \psi)$. Hence

$$
|W(F ; \psi)| \geq|U| \geq 1-\varepsilon
$$

and since $\varepsilon$ was arbitrary, we are done.

Combining Lemmas 2.4, 2.5 and 2.12 proves Theorem 2.2 ,

2.4. Missing cases. Despite having dealt with a very large class of distance functions in the preceding two sections, there are still two cases not covered by our results. The first is when $\operatorname{skel}(F)$ consists of infinitely many rationally sloped lines, when the notion of a fundamental region makes little sense. We conjecture that this case may be treated analogously with the irrational case. This should be possible as the resonant sets will induce geodesics on the torus winding around arbitrarily many times.

The second is when each irrationally sloped lines of skel $(F)$ is insignificant. We conjecture that these may be removed from the star body without affecting the corresponding Khintchine type result, which reduces to the rational case. While these conjectures seem reasonable to us, we have not yet been able to prove them. 


\section{TRANSFERENCE PRINCIPLES}

In the rational case, it makes sense to talk about transference principles. We will give a direct proof of Theorem 1.1] and then give a description of how this method may be extended to other star bodies where the skeleton consists of lines with rational slopes. We prove the theorem in arbitrary dimension, as this causes no loss of clarity. In addition to the proof of Theorem 1.1, we illustrate the versatility of our method by sketching a proof of a transference principle for a distance function not covered by any previous results.

We first prove some preliminary equivalences.

Proposition 3.1. Let the distance function $F: \mathbb{R}^{n} \rightarrow[0, \infty)$ be given by

$$
F(x)=F\left(x_{1}, \ldots, x_{n}\right)=\left(\prod_{i=1}^{n}\left|x_{i}\right|\right)^{1 / n}
$$

and define the distance function $H_{\nu}: \mathbb{R}^{n} \rightarrow[0, \infty)$ by

$$
H_{\nu}(x)=\max \left\{\nu_{1}\left|x_{1}\right|, \ldots, \nu_{n}\left|x_{n}\right|\right\},
$$

where $\nu=\left(\nu_{1}, \ldots, \nu_{n}\right) \in(0, \infty)^{n}$. Then for each $\lambda>0, F(x) \leq \lambda$ if and only if $H_{\nu}(x) \leq \lambda$ for some $\nu$ with $\nu_{1} \cdots \nu_{n}=1$.

Both functions in Proposition 3.1 are distance functions which are symmetric about the origin (a planar configuration is shown in Figure 2).

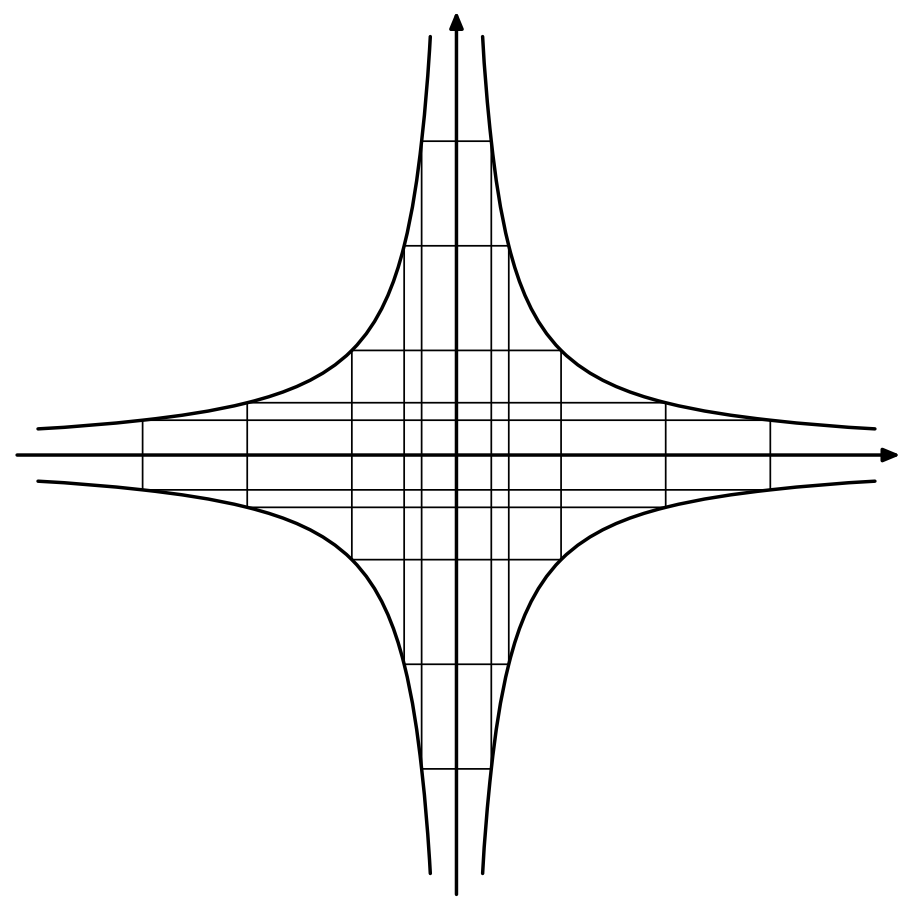

FiguRE 2. The star body corresponding to $F$ for $n=2$ with some parametrising boxes. 
Proof. To begin, assume that $\left|x_{1}\right| \cdots\left|x_{n}\right| \leq \lambda^{n}$. We will find $\nu_{1}, \ldots, \nu_{n}$ with the required properties. For $i=1, \ldots, n-1$, let $\nu_{i}=\lambda /\left|x_{i}\right|$ and let $\nu_{n}=1 /\left(\nu_{1} \cdots \nu_{n-1}\right)$. We only need to prove that $\nu_{n}\left|x_{n}\right| \leq \lambda$. But this is the case, since by assumption

$$
\nu_{n}\left|x_{n}\right|=\frac{\left|x_{1}\right| \cdots\left|x_{n}\right|}{\lambda^{n-1}} \leq \frac{\lambda^{n}}{\lambda^{n-1}}=\lambda .
$$

Then converse is even easier, since

$$
\left|x_{1}\right| \cdots\left|x_{n}\right|=\nu_{1}\left|x_{1}\right| \cdots \nu_{n}\left|x_{n}\right| \leq \lambda^{n}
$$

by assumption.

Proposition 3.2. Let $\mu, \lambda>0$. The following are equivalent:

(i) There is an integer solution $q \in \mathbb{Z}^{n} \backslash\{0\}$ to

$$
|\langle q \cdot x\rangle| \leq \lambda, \quad\left(\prod_{i=1}^{n} \max \left(\left|q_{i}\right|, 1\right)\right)^{1 / n} \leq \mu .
$$

(ii) There is an integer solution $p \in \mathbb{Z} \backslash\{0\}$ to

$$
\left(\prod_{i=1}^{n}\left|\left\langle p x_{i}\right\rangle\right|\right)^{1 / n} \leq n \lambda, \quad|p| \leq n \mu \lambda^{(1-n) / n} .
$$

Proof. Using Proposition 3.1, we may encode solutions to these equations in matrices as follows. Define the matrices

$$
\begin{aligned}
A & =\left(\begin{array}{cccc}
\lambda^{-1} x_{1} & \nu_{1} \mu^{-1} & \ldots & 0 \\
\vdots & \vdots & \ddots & \vdots \\
\lambda^{-1} x_{n} & 0 & \ldots & \nu_{n} \mu^{-1} \\
\lambda^{-1} & 0 & \ldots & 0
\end{array}\right), \\
A^{*} & =\left(\begin{array}{cccc}
\lambda & -\mu \nu_{1}^{-1} x_{1} & \ldots & -\mu \nu_{n}^{-1} x_{n} \\
0 & \mu \nu_{1}^{-1} & \ldots & 0 \\
\vdots & \vdots & \ddots & \vdots \\
0 & 0 & \ldots & \mu \nu_{n}^{-1}
\end{array}\right) .
\end{aligned}
$$

If (23) has a non-trivial integer solution $q=\left(q_{1}, \ldots, q_{n}\right)$, then for some choice of $\nu_{1}, \ldots, \nu_{n}$ with $\nu_{1} \cdots \nu_{n}=1$, then the inequality

$$
|\tilde{q} A|_{\infty} \leq 1
$$

has a non-trivial integer solution $\tilde{q}=\left(q_{1}, \ldots, q_{n}, p\right)$. This follows on considering each coordinate of $\tilde{q} A$ and applying Proposition 3.1 .

As can be verified by calculation, when $(\tilde{a}, \tilde{b}) \in \mathbb{Z}^{n+1} \times \mathbb{Z}^{n+1}$, the linear form

$$
\Phi(\tilde{a}, \tilde{b})=\tilde{a} A \cdot \tilde{b} A^{*}=a_{1} b_{2}+\cdots+a_{n} b_{n+1}+a_{n+1} b_{1} \in \mathbb{Z}
$$

when $\tilde{x}, \tilde{y}$ are integer vectors. By (26) and [7, Chapter V, Theorem I], there is an integer solution to

$$
\left|\tilde{p} A^{*}\right| \leq n\left|\operatorname{det}\left(A^{*}\right)\right|^{1 / n}=n \mu \lambda^{1 / n}
$$


since $\nu_{1}^{-1} \cdots \nu_{n}^{-1}=\left(\nu_{1} \cdots \nu_{n}\right)^{-1}=1$. Again by Proposition 3.1. (24) has a non-trivial integer solution. By the same method we find that if (24) has a non-trivial solution, then (23) has a non-trivial solution.

Proof of Theorem 1.1. Assume that condition (i) of the theorem holds. Let $q^{(j)}$ be the sequence of solutions to inequality (41) and order these such that $j^{\prime}>j$ implies that $F_{+}\left(q^{\left(j^{\prime}\right)}\right) \geq F_{+}\left(q^{(j)}\right)$, where

$$
F_{+}(x)=\left(\prod_{j=1}^{n} \max \left\{1,\left|x_{j}\right|\right\}\right)^{1 / n} .
$$

Furthermore, since equality holds at most finitely often, and since there are infinitely many solutions, $F_{+}\left(q^{(j)}\right)$ tends to infinity as $j$ tends to infinity. Now for each $j \in \mathbb{N}$, define numbers

$$
\mu_{j}=F_{+}\left(q^{(j)}\right), \quad \lambda_{j}=\mu_{j}^{-1-\varepsilon} .
$$

With these coefficients, (23) has $q^{(j)}$ as a solution for each $j \in \mathbb{N}$. By Proposition 3.2. (24) has a non-trivial integer solution $p^{(j)}$ for each $j \in \mathbb{N}$. Let $j$ be fixed but arbitrary. For ease of notation, we drop the index in the following.

From (24),

$$
F(\langle p a\rangle) \leq n \lambda, \quad|p| \leq n \mu \lambda^{(1-n) / n} .
$$

Let $\varepsilon^{\prime} \in(0, \varepsilon)$ be fixed but arbitrary. To prove that there is a solution to (5) of condition (ii), the $\lambda$ and $\mu$ are eliminated. The second inequality immediately implies that

$$
\begin{aligned}
|p|^{-\left(1+\varepsilon^{\prime}\right) / n} & \geq\left(n \mu \lambda^{(1-n) / n}\right)^{-\left(1+\varepsilon^{\prime}\right) / n} \\
& =\left(n^{-(1 / n)-\left(\varepsilon^{\prime} / n\right)-1} \mu^{-(1 / n)-\left(\varepsilon^{\prime} / n\right)} \lambda^{-\left(1 / n^{2}\right)+(1 /) n-\left(\varepsilon^{\prime} / n^{2}\right)+\left(\varepsilon^{\prime} / n\right)-1}\right) n \lambda .
\end{aligned}
$$

Hence, if we can prove that $\varepsilon^{\prime}$ and $j$ may be chosen so that the term in the brackets is greater than or equal to 1 , this implies that we have a solution to inequality (5). We insert the definition of $\lambda$ in (27) to see that this is equivalent to the condition that

$$
n^{-(1 / n)-\left(\varepsilon^{\prime} / n\right)-1} \mu^{-(2 / n)-\left(2 \varepsilon^{\prime} / n\right)+\left(1 / n^{2}\right)+\left(\varepsilon^{\prime} / n^{2}\right)+1+\left(\varepsilon / n^{2}\right)-(\varepsilon / n)+\left(\varepsilon \varepsilon^{\prime} / n^{2}\right)-\left(\varepsilon \varepsilon^{\prime} / n\right)+\varepsilon} \geq 1 .
$$

Using the fact that $\mu$ is increasing and unbounded as a function of $j$ and on choosing $\varepsilon^{\prime}$ sufficiently small, it is straightforward to verify the claim.

To prove the first implication, we need to show that this produces infinitely many solutions to inequality (5). But again, this follows from Proposition 3.2. Indeed, assume that we have only solutions $p$ to (24) with bounded height. Then there are only solutions $q$ with bounded $F(q)$, and hence only finitely many solutions.

The converse implication is proved analogously by interchanging the two inequalities in the above.

The above proof can be generalised to other star bodies than the multiplicative one. Indeed, we have only used the fact that solutions to the relevant inequalities may be encoded in matrix form (25), parametrised by the numbers $\nu_{i}$. This parametrisation in turn depends only on exhibiting an appropriate cover of the star body corresponding to $F$. In the case studied above, the star body is covered by boxes with sides parallel to the 
coordinate axes (see figure 21). We now give an example when this is not the case. For convenience, we restrict ourselves to the planar case.

The 'union jack' star body is obtained by taking a union of the multiplicative star body and a rotation of it through $\pi / 4$. The associated distance function is given by

$$
F^{\prime}(x, y)=\min \left\{|x y|, \frac{\left|x^{2}-y^{2}\right|}{2}\right\}^{1 / 2} .
$$

A plot of the star body is given in figure 3. For this distance function, the above

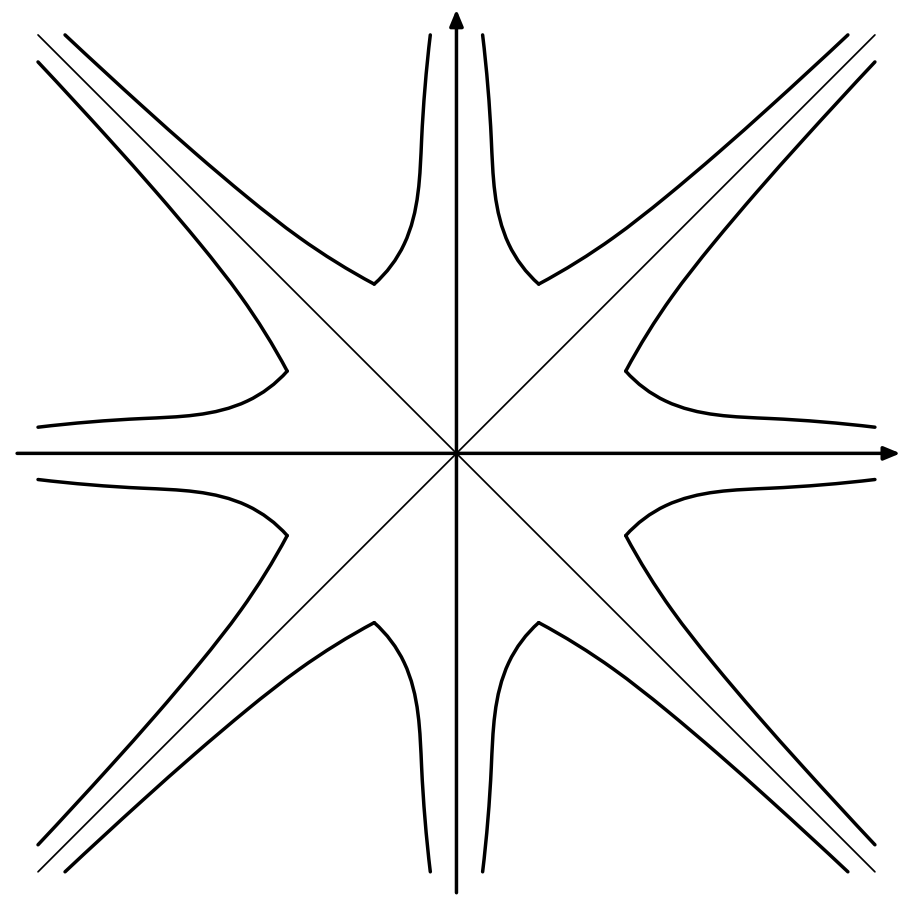

Figure 3. The 'union jack' star body

argument gives the following transference principle.

Theorem 3.3. Let $(x, y) \in(\mathbb{R} \backslash \mathbb{Q})^{2}$. The following two conditions are equivalent

(i) For some $\varepsilon>0$, there are infinitely many $q_{1}, q_{2} \in \mathbb{Z}$ such that

$$
\begin{aligned}
\left|\left\langle q_{1} x+q_{2} y\right\rangle\right| \leq \min \left\{\max \left\{\left|q_{1}\right|, 1\right\} \max \left\{\left|q_{2}\right|, 1\right\}\right. \\
\left.\qquad \frac{\sqrt{2}}{2} \max \left\{\left|q_{1}+q_{2}\right|, 1\right\} \max \left\{\left|q_{1}-q_{2}\right|, 1\right\}\right\}^{-1-\varepsilon} .
\end{aligned}
$$

(ii) For some $\varepsilon^{\prime}>0$, there are infinitely many $p \in \mathbb{Z}$ such that

$$
F^{\prime}(\langle(p x, p y)\rangle) \leq|p|^{-\left(1+\varepsilon^{\prime}\right) / 2}
$$


The proof is essentially the same, except that instead of matrices $A$ and $A^{*}$ from (25), we use four matrices

$$
\begin{aligned}
& \tilde{A}=\left(\begin{array}{ccc}
\lambda^{-1} x & \nu_{1} \mu^{-1} & 0 \\
\lambda^{-1} y & 0 & \nu_{2} \mu^{-1} \\
\lambda^{-1} & 0 & 0
\end{array}\right), \quad \tilde{A}^{\prime}=\left(\begin{array}{ccc}
\lambda^{-1} x & \frac{\sqrt{2}}{2} \nu_{1}^{\prime} \mu^{-1} & -\frac{\sqrt{2}}{2} \nu_{2}^{\prime} \mu^{-1} \\
-\lambda^{-1} y & \frac{\sqrt{2}}{2} \nu_{1}^{\prime} \mu^{-1} & \frac{\sqrt{2}}{2} \nu_{2} \mu^{-1} \\
\lambda^{-1} & 0 & 0
\end{array}\right), \\
& \tilde{\tilde{A}}=\left(\begin{array}{ccc}
\lambda & -\mu \nu_{1}^{-1} x & -\mu \nu_{2}^{-1} y \\
0 & \mu \nu_{1}^{-1} & 0 \\
0 & 0 & \mu \nu_{2}^{-1}
\end{array}\right), \quad \tilde{\tilde{A}}^{\prime}=\left(\begin{array}{ccc}
\lambda & -\frac{\sqrt{2}}{2} \mu \nu_{1}^{\prime-1}(x-y) & \frac{\sqrt{2}}{2} \mu \nu_{2}^{\prime-1}(x+y) \\
0 & \frac{\sqrt{2}}{2} \mu \nu_{1}^{\prime-1} & \frac{\sqrt{2}}{2} \mu \nu_{2}^{\prime-1} \\
0 & -\frac{\sqrt{2}}{2} \mu \nu_{1}^{\prime-1} & \frac{\sqrt{2}}{2} \mu \nu_{2}^{\prime-1}
\end{array}\right),
\end{aligned}
$$

parametrised by $\nu_{1}, \nu_{2}, \nu_{1}^{\prime}, \nu_{2}^{\prime}>0$, where $\nu_{1} \nu_{2}=\nu_{1}^{\prime} \nu_{2}^{\prime}=1$. It is easily checked that these matrices encode solutions to the inequalities corresponding to (23) and (24) in the present setting and so provide an analogue of Proposition 3.2. i.e., we now look at integer solutions to

and similarly to

$$
\min \left\{|\tilde{q} \tilde{A}|_{\infty},\left|\tilde{q} \tilde{A}^{\prime}\right|_{\infty}\right\} \leq 1
$$

$$
\min \left\{|\tilde{p} \tilde{\tilde{A}}|_{\infty},\left|\tilde{q} \tilde{\tilde{A}}^{\prime}\right|_{\infty}\right\} \leq 1
$$

The proof of Theorem 3.3 is now analogous to that of Theorem 1.1. We leave details to the reader.

Clearly, this approach can be generalised to other star bodies, where the parametrisation depends on the distance function, and so the above proof can be seen as a recipe for proving transference principles. However, apart from the minor variations of standard known cases of simultaneous (height), dual (inner product) and multiplicative approximation (see e.g. [9, 11]), it does not appear that there is a simple general transference principle for arbitrary distance functions.

\section{ACKNOWLEDGEMENTS}

We thank Victor Beresnevich for pointing out an error in an earlier version of the paper and Yann Bugeaud for drawing our attention to Dyson's paper 11. We are grateful for support from EPSRC (grant no. GR/N02832/01) and INTAS (grant no. 001-429). SK is a William Gordon Seggie Brown Fellow.

\section{REFERENCES}

[1] I. Aliev and P. M. Gruber, Lattice points in large Borel sets and successive minima, to appear in Discrete Comput. Geom.

[2] A. Baker, On some Diophantine inequalities involving the exponential function, Canad. J. Math. 17 (1965). 616-626.

[3] V. Beresnevich, D. Dickinson, and S. L. Velani, Measure theoretic laws for lim sup sets, to appear in Mem. Amer. Math. Soc.

[4] V. Bernik, D. Kleinbock, and G. A. Margulis, Khintchine-type theorems on manifolds: the convergence case for standard and multiplicative versions, Internat. Math. Res. Notices (2001), no. 9, $453-486$.

[5] L. Breiman, Probability, Classics in Applied Mathematics, vol. 7, Society for Industrial and Applied Mathematics (SIAM), Philadelphia, PA, 1992, Corrected reprint of the 1968 original.

[6] Y. Bugeaud, A note on inhomogeneous Diophantine approximation, Glasg. Math. J. 45 (2003), no. $1,105-110$. 
[7] J. W. S. Cassels, An introduction to Diophantine approximation, Cambridge Tracts in Mathematics and Mathematical Physics, No. 45, Cambridge University Press, New York, 1957.

[8] J. W. S. Cassels, An introduction to the geometry of numbers, Die Grundlehren der mathematischen Wissenschaften in Einzeldarstellungen mit besonderer Berücksichtigung der Anwendungsgebiete, Bd. 99 Springer-Verlag, Berlin-Göttingen-Heidelberg, 1959.

[9] M. M. Dodson, Star bodies and Diophantine approximation, J. London Math. Soc. (2) 44 (1991), no. $1,1-8$.

[10] M. M. Dodson and S. Hasan, Systems of linear forms and covers for star bodies, Acta Arith. 61 (1992), no. 2, 119-127.

[11] F. J. Dyson, On simultaneous Diophantine approximations, Proc. London Math. Soc. (2) 49 (1947), 409-420.

[12] P. Gallagher, Metric simultaneous diophantine approximation, J. London Math. Soc. 37 (1962), 387-390.

[13] A. Katok and B. Hasselblatt, Introduction to the modern theory of dynamical systems, Encyclopedia of Mathematics and its Applications, vol. 54, Cambridge University Press, Cambridge, 1995.

[14] D. Y. Kleinbock and G. A. Margulis, Flows on homogeneous spaces and Diophantine approximation on manifolds, Ann. of Math. (2) 148 (1998), no. 1, 339-360.

[15] S. Kristensen, Metric diophantine approximation with respect to planar distance functions, to appear in The Proceedings of the Institute of Mathematics NAN Belarus.

[16] J. Schmeling and S. Trubetskoŭ, Inhomogeneous Diophantine approximations and angular recurrence for billiards in polygons, Mat. Sb. 194 (2003), no. 2, 129-144.

[17] V. T. Sós, On the theory of diophantine approximations. I, Acta Math. Acad. Sci. Hungar 8 (1957), 461-472.

[18] V. G. Sprindžuk, Metric theory of Diophantine approximations, V. H. Winston \& Sons, Washington, D.C., 1979.

[19] Y. Wang and K. R. Yu and Y. C. Zhu, A transfer theorem for linear forms (in Chinese), Acta Math. Sinica 22 (1979), 237-240.

M. M. Dodson, Department of Mathematics, University of York Heslington, York, YO10 5DD, UNITED KINGDOM

E-mail address: mmd1@york.ac.uk

S. Kristensen, School of Mathematics, University of Edinburgh, James Clerk Maxwell Building, King's Buildings, Mayfield RoAd, Edinburgh, EH9 3JZ, United Kingdom

Current address: Department of Mathematical Sciences, Faculty of Science, University of Aarhus, Ny

Munkegade, Building 530, DK-8000 Aarhus C, Denmark

E-mail address: sik@imf .au.dk 